\title{
Genomic basis of ecological niche divergence among cryptic sister species of non-biting midges
}

\author{
Hanno Schmidt ${ }^{1,2}$, Bastian Greshake ${ }^{1}$, Barbara Feldmeyer ${ }^{1,3}$, Thomas Hankeln ${ }^{2}$ and Markus Pfenninger ${ }^{1 *}$
}

\begin{abstract}
Background: There is a lack of understanding the evolutionary forces driving niche segregation of closely related organisms. In addition, pinpointing the genes driving ecological divergence is a key goal in molecular ecology. Here, larval transcriptome sequences obtained by next-generation-sequencing are used to address these issues in a morphologically cryptic sister species pair of non-biting midges (Chironomus riparius and C. piger).

Results: More than eight thousand orthologous open reading frames were screened for interspecific divergence and intraspecific polymorphisms. Despite a small mean sequence divergence of $1.53 \%$ between the sister species, $25.1 \%$ of 18,115 observed amino acid substitutions were inferred by a statistics to be driven by positive selection. Applying McDonald-Kreitman tests to 715 alignments of gene orthologues identified eleven (1.5\%) genes driven by positive selection.

Conclusions: Three candidate genes were identified as potentially responsible for the observed niche segregation concerning nitrite concentration, habitat temperature and water conductivity. Additionally, signs of positive selection in the hydrogen sulfide detoxification pathway were detected, providing a new plausible hypothesis for the species' ecological differentiation. Finally, a divergently selected, nuclear encoded mitochondrial ribosomal protein may contribute to reproductive isolation due to cytonuclear coevolution.
\end{abstract}

Keywords: Adaptive sequence evolution, Positive selection, McDonald-Kreitman test, Chironomus riparius, Chironomus piger

\section{Background}

A decade-long debate reasons to what extent Darwinian selection or neutral processes are driving the molecular evolution of genes and thus the ecological divergence of species [1-4]. Selectionists argue that a large fraction of those non-synonymous DNA base substitutions in coding genes going to fixation should be driven by positive Darwinian selection [5]. Under a strict neutralist's view, most fixed amino acid substitutions have no effect on fitness [6,7], because purifying selection constantly removes alleles with strongly deleterious effects on fitness while positive effects were thought to be extremely rare. Later, the nearly neutral theory acknowledged that also substitutions with slightly deleterious effects may drift to fixation under realistic demographic scenarios

\footnotetext{
* Correspondence: pfenninger@bio.uni-frankfurt.de

'Molecular Ecology Group, Biodiversity and Climate Research Centre (BiK-F) by Senckenberg Gesellschaft für Naturforschung and Goethe University, Biocampus Siesmayerstraße, 60054, Frankfurt am Main, Germany Full list of author information is available at the end of the article
}

$[8,9]$. While the neutral theory was the prevailing model for several decades, the comparison of whole genome sequences has recently produced evidence for an important role of natural selection [3]. In particular, for Drosophila species, natural selection has been shown to shape both the coding and non-coding parts of the genome $[10,11]$. However, before being able to draw general conclusions on the importance and mode of selection in shaping ecological divergence, more studies of systematically diverse taxa with differing life histories, demographies and mating systems are clearly needed [3]. The recent progress in sequencing technology $[12,13]$ and resultant ability to sequence whole transcriptomes or genomes even for non-model species now opens up this opportunity [14]. Moreover, such approaches allow at the same time pinpointing the genomic basis of ecologically relevant traits and their evolutionary history [15-19]. This may be accomplished in two different ways: Based on known ecological differences of the taxa under scrutiny it is possible to assess the processes 
driving the evolution of genes likely associated with the relevant traits. This is an extension of the classical candidate gene or top down approach. In addition, scanning coding genes for positive selection allows for a bottom up approach, which Li et al. [20] called "reverse ecology". The principle of the latter is to identify loci whose divergence was driven by positive selection and to infer hypotheses about ecological differences from their biological function. In both cases, however, it remains challenging to functionally link the identified patterns with observed phenotypic differences.

To contribute to this scientific debate, we have conducted a comparative analysis of larval transcriptomes among the dipteran midge sister species pair Chironomus riparius Meigen 1804 (synonym C. thummi, respectively C. thummi thummi) and Chironomus piger Strenzke 1959 (synonym C. thummi piger) [21]. As co-occurring, morphologically cryptic sister species, they are particularly interesting to perform comparative genomic analyses of ecological niche differences for several reasons. First, due to the shared evolutionary history, their general ecological niche is usually similar, which makes them prime candidates for interspecific competition and, to allow coexistence in sympatry, niche segregation [22]. Second, fixed genetic differences among them must have evolved during or after divergence, thereby also reflecting the selective forces leading to the observed ecological differences and/ or reproductive isolation. Third, their coding gene sequences will almost certainly be sufficiently similar to distinguish orthologous from paralogous loci [23]. Fourth, the short evolutionary distance assures that the incidence of multiple mutational hits at individual sites is negligible, making it possible to infer which mutational changes have occurred since speciation [24]. Fifth, as morphologically cryptic species, identified differences likely not involve anatomic differences, thereby reducing the complexity of associating gene evolution with phenotypic differences.

The two Chironomus species show differential swarming behaviour in the field, acting as a prezygotic isolation mechanism [25]. While some studies indicate that $C$. riparius and $C$. piger readily form viable and fully fertile interspecific hybrids in the laboratory [26], others estimate fertile hybrids in the wild to be effectively absent, due to fertility reductions caused by hybrid dysgenesis syndromes [27]. Indeed, no ongoing hybridisation in the field could be found in an early chromosomal study [28], which was corroborated by a field survey applying microsatellites and mitochondrial markers [29]. Larvae of both species are widely distributed in small streams, ditches, ponds and puddles throughout the Holarctic [28]. The species are often dominating the local Chironomus larval community [30]. They frequently occur together at the same sites, however, usually one species prevails at a particular site [28,29]. As chironomids spend most of their lifetime as larvae and usually even do not feed as imagines during their few days in this stage [31], the larval stage is in general regarded as the relevant life cycle stage for ecological studies [32]. This is especially true for the two species under scrutiny, $C$. piger and C. riparius, as they show clear ecological differentiation as larvae [29] but nearly none as imagines [25]. Field data indicate that C. piger larvae are found preferentially in puddles and shallow ditches with higher maximum water temperatures, higher salinity, in particular higher nitrite and calcium concentrations as compared to sites inhabited by C. riparius [29]. The latter species often inhabits sediments with high organic content, indicating higher tolerance to anaerobic conditions [33], which was confirmed in different experimental studies [34,35]. In a recent experimental study $C$. piger coped better with higher nitrite concentrations [36]. Contrary to expectations from field studies, $C$. riparius' fitness tended to be higher at both higher constant temperatures and larger daily temperature ranges. However, the interaction of both stressors favoured $C$. piger in warm, high nitrite habitats, thus concurring to the field observations [36]. Based on this previous knowledge on ecological niche differences, proteins with functions in cell respiration as well as those concerning response to temperature and solved ions, especially nitrite detoxification, are promising candidates for interspecific differences driven by positive selection.

In this study, the following three questions were thus addressed: i) Is positive Darwinian selection a major evolutionary driving force for the divergence of the sister species? ii) Can genes with signs of positive selection be conclusively linked to known ecological differences between the two sister species? iii) Can we derive hypotheses on yet unrecognised ecological differences between the sister species from the observed pattern of divergence?

\section{Results}

\section{Sequencing, assembly and annotation}

Sequencing the larval transcriptomes of the two midge species with the Roche 454 technique resulted in $2,123,605$ quality filtered reads in total (Table 1). Assembly using the CLC Genomics Workbench yielded 42,524.

Table 1 Summary statistics of transcriptome sequencing

\begin{tabular}{lll}
\hline & C. piger & C. riparius \\
\hline Number of 454 reads & $1,235,393$ & 888,212 \\
Number of contigs & 42,524 & 29,917 \\
Contig N50 length (bp) & 805 & 914 \\
Number of BLASTx hits $<1 e^{-10}$ & 11,326 & 9,187 \\
Number of unigenes & 6,323 & 5,705 \\
\hline
\end{tabular}


contigs for $C$. piger and 29,917 for $C$. riparius, respectively (Table 1 ). Mean contig length was 621 bp for $C$. piger and 732 bp for $C$. riparius. N50 length was 805 bp for $C$. piger and $914 \mathrm{bp}$ for $C$. riparius. Ninety-three percent (C. piger) and $94 \%$ (C. riparius) of all reads could be mapped against the obtained contigs.

Using the BLASTx algorithm, 11,326 contigs of $C$. piger and 9,187 contigs of $C$. riparius matched entries in the Swiss-Prot database with e-values below the threshold of $1 \mathrm{e}^{-10}$. Merging hits with identical descriptions irrespective of the hits' taxon assignment resulted in 6,323 unigenes in $C$. piger and 5,705 unigenes in $C$. riparius with an overlap of 4,738 genes. Of all contigs with BLASTx hits below $1 \mathrm{e}^{-10}$ a total of 9,527 could be annotated with GO terms (Additional file 1).

\section{Alignment quality and sequence divergence}

The programme ORFPredictor assigned open reading frames (ORFs), matching the set requirements, for 41,489 contigs in C. piger (98\%) and for 29,391 contigs in C. riparius (98\%), respectively. OrthoMCL built 12,685 groups of putative orthologues from translated sequences of the two sets of ORFs from C. piger and $C$. riparius. As 2,302 groups only contained sequences from one or the other species, a total of 10,383 groups were kept. For each group the best-fitting sequences of the two species were then aligned and re-translated. Trimming of those alignments ended in 8,031 final alignments with an average length of $402 \mathrm{bp}$. Of those, 1,711 alignments (21.3\%) had no differences on the nucleotide level. Besides that, there was an apparent peak around $1 \%$ sequence divergence between the species (Figure 1). The mean sequence divergence was $1.53 \%$ on the nucleotide level and $1.68 \%$ on the amino acid level, equalling 18,115 amino acid substitutions in total.

\section{Detection of intraspecific genetic variation}

Single nucleotide polymorphism (SNP) identification in the species-specific mappings considered sequencing quality scores of the used and surrounding nucleotides, mapping depth and quantity of the rarer allele for quality control. SNP detection across the 42,524 contigs in C. piger identified 72,096 high quality diallelic SNP positions. After discarding all SNPs outside the defined high quality ORFs, 25,375 SNP sites remained. Of those, 13,434 (52.9\%) were non-silent, and 11,941 (47.1\%) were silent. The 29,917 contigs in C. riparius yielded 33,826 SNP positions in total, 12,032 of which were within a high quality ORF. At 6,720 SNP positions (55.9\%) the two alleles coded for different amino acids, while 5,312 SNP sites (44.2\%) were silent. Classification of SNPs according to the frequency of their rarer allele showed the ratio of non-synonymous to synonymous SNPs to decrease at higher allele frequency (Table 2).

\section{Measure of sequence evolution for the whole transcriptome}

The estimated average proportion of amino acid substitutions fixed by positive selection, $\alpha$, amounted to 0.251 for the 8,031 alignments included. This estimate is significantly greater than zero with regard to the calculated confidence interval (95\% C. I.: 0.192, 0.308). The analysis was repeated under exclusion of (1) low-frequency and (2) low+moderate-frequency SNPs to correct for slightly deleterious mutations. This procedure slightly elevated $\alpha$ to (1) 0.265 (C. I.: 0.206, 0.323) and (2) 0.324 (C. I.: $0.264,0.378)$, respectively.

\section{McDonald-Kreitman tests}

The McDonald-Kreitman test (MKT), a robust test for positive selection using substitution and polymorphism data, could be applied to 715 alignments showing nonzero values in all four categories of nucleotide changes. Of those alignments, 11 (1.5\%) had a ratio of nonsynonymous substitutions $\left(D_{n}\right)$ to synonymous substitutions $\left(D_{s}\right)$ significantly greater than the ratio of nonsynonymous polymorphisms $\left(\mathrm{P}_{\mathrm{n}}\right)$ to synonymous polymorphisms $\left(\mathrm{P}_{\mathrm{s}}\right)$ with a FDR-corrected p-value $\leq 0.05$, hence bore signs of positive selection between $C$. piger and $C$. riparius. Due to lacking BLASTx annotations in three of them, eight alignments with reliable signs of positive selection and available annotations remained (Table 3).

\section{Branch-specific $\omega$}

OrthoMCL built 19,800 groups of putative orthologous sequences by means of the four sets of protein sequences from $C$. piger, $C$. riparius and the two mosquitoes $A$. aegypti and $C$. quinquefasciatus. As only groups with at least one sequence per species were analysed further, a total of 4,232 groups were kept. Trimming of the re-translated alignments of the best-fitting sequences per species resulted in 2,558 final alignments with an average length of $496 \mathrm{bp}$. Using the programme PAML, signs of positive selection were found in 316 alignments (12\%) in $C$. piger and 336 alignments (13\%) in C. riparius.

Among the eight annotated genes with signs of positive selection in the MKT, two had branch-specific $\omega>1$ in one species and $\omega<1$ in the other, indicating directional selection. Here, plasma membrane calciumtransporting ATPase 3 showed signs of positive selection only in $C$. piger and mitochondrial sulfide:quinone oxidoreductase only in C. riparius. One protein (39S ribosomal protein L44, mitochondrial) showed $\omega$ larger than one in both species, indicating disruptive selection between the species. The rest were either incalculable in one or both species due to missing sequence data in the four-species alignments $(4 \times)$ or had branch-specific $\omega$ smaller than one $(1 \times)$. 


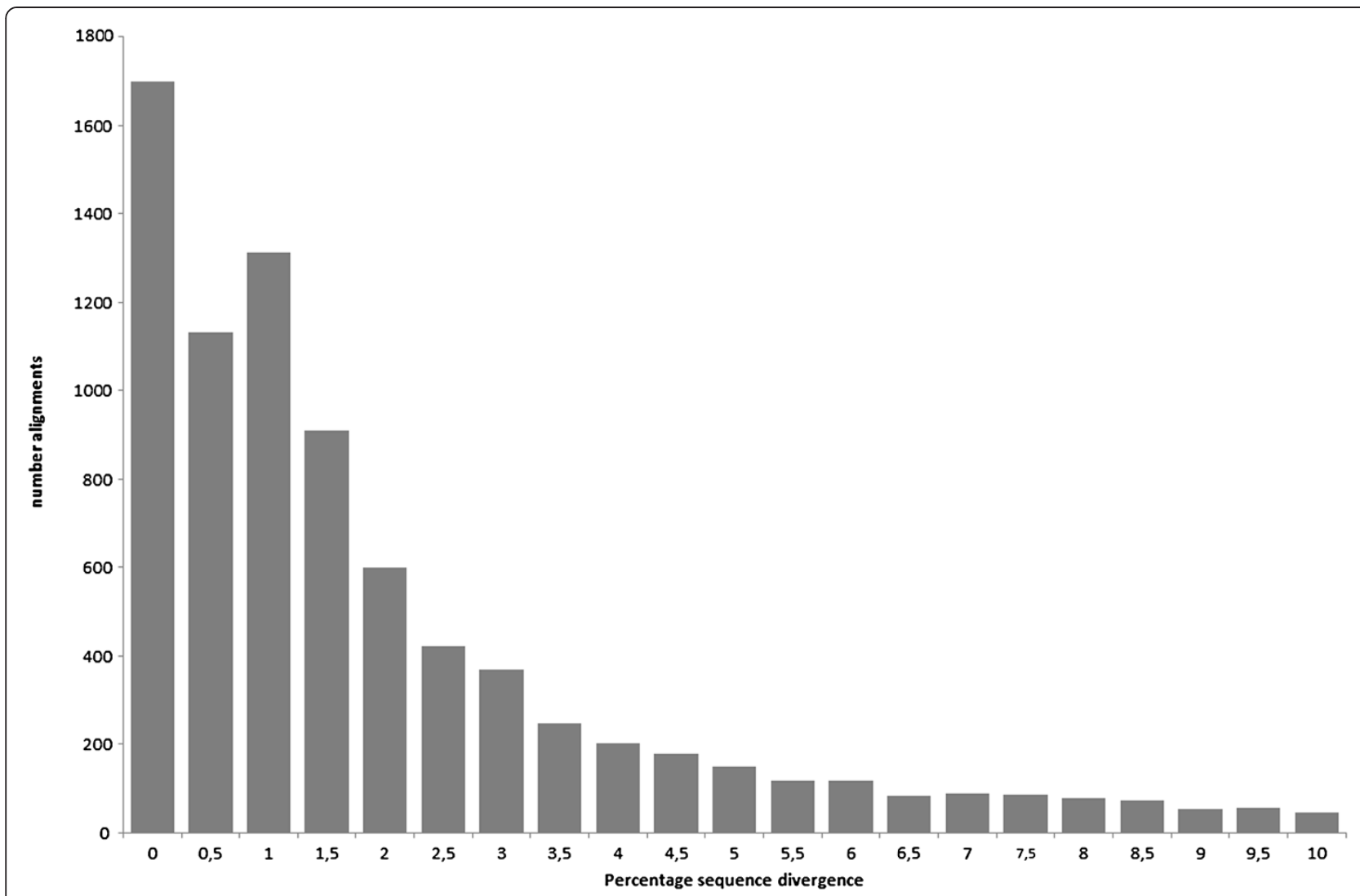

Figure 1 Distribution of uncorrected sequence divergence between transcribed genes of $\boldsymbol{C}$. piger and $\boldsymbol{C}$. riparius. Shown are the rates of substitutions between the two chironomid species per alignment. Alignments with more than $10 \%$ sequence divergence were not taken into account.

\section{Discussion}

Comparative sequence-based studies are highly dependent on data quality. The stringent approach taken here with orthology assignment of high quality contigs and rigorous trimming of the resultant alignments is therefore rather conservative, despite a considerable loss of data. One source of errors that necessitated substantial trimming of the data was homopolymer stretches that pose well-known problems when sequencing with the 454 Roche technology $[12,37,38]$. By only using SNPs

Table 2 Number and frequency distribution of SNPs in the two sister species

\begin{tabular}{llll}
\hline & Pn & Ps & Pn*/Ps* \\
\hline Low frequency SNPs & 2,421 & 1,341 & 0.46 \\
Moderate frequency SNPs & 4,069 & 3,326 & 0.31 \\
Common SNPs & 13,666 & 12,586 & 0.28 \\
\hline
\end{tabular}

SNPs were classified by the frequency of their rarer allele as follows: low frequency $<5 \%$, moderate frequency $5-15 \%$, and common $15-50 \%$. Pn $=$ nonsynonymous SNPs, Ps = synonymous SNPs, $\mathrm{Pn}^{*} / \mathrm{Ps}^{*}=$ ratio of nonsynonymous to synonymous SNPs per non-synonymous and synonymous sites, respectively. with the rarer allele observed at least twice, a misidentification of SNPs due to sequencing errors should not have posed considerable problems as the overall sequencing error rate (including both, indels and base substitutions) is only $1.07 \%$ for 454 GS FLX [39]. With a mean coverage of 176 observed at the SNP positions used, this equals $\mathrm{p}=0.02$. Considering that only a small portion of those 454 sequencing mistakes are base replacement errors [39], the effective error rate is even lower. Furthermore, included errors will most certainly contribute proportionally more to non-synonymous than to synonymous SNPs due to the high ratio of non-synonymous to synonymous positions. The resulting overestimation of the ratio of non-synonymous to synonymous SNPs would therefore rather lead to a lowered, conservative signal of the impact of adaptive evolution in the MKT and the calculation of $\alpha$.

It is, however, important to be aware of other immanent limitations of Next-Generation-Sequencing-generated transcriptomic data for population genetic analyses. Transcriptomic sequences might miss alleles due to allele specific gene expression [40]. This phenomenon is 
Table 3 Alignments with significant MKT values and their correlating $\omega$ values

\begin{tabular}{|c|c|c|c|c|c|c|c|}
\hline Cluster & BLASTx annotation & $\begin{array}{l}(\mathrm{Dn} / \mathrm{Ds}) / \\
(\mathrm{Pn} / \mathrm{Ps})\end{array}$ & $\omega$ interspecific & $\begin{array}{l}\omega \\
\text { C. piger }\end{array}$ & $\begin{array}{l}\omega \\
\text { C. riparius }\end{array}$ & GO terms Biological Process & $\begin{array}{l}\text { Hypothesised relation } \\
\text { to observed niche } \\
\text { differences }\end{array}$ \\
\hline rp4125 & $\begin{array}{l}\text { Plasma membrane calcium- } \\
\text { transporting ATPase } 3\end{array}$ & 9.24 & 0.12 & 9.75 & 0.08 & $\begin{array}{l}\text { metabolic process, calcium ion } \\
\text { transmembrane transport, blood } \\
\text { coagulation, ATP biosynthetic } \\
\text { process, platelet activation }\end{array}$ & $\begin{array}{l}\text { Tolerance of } \mathrm{C} \text {. piger to } \\
\text { increased } \mathrm{CaCO}_{3} \text { levels due } \\
\text { to effective removal of } \\
\text { Ca-ions from cells. }\end{array}$ \\
\hline rp4927 & $\begin{array}{l}\text { Dolichyl-diphosphooligosaccharide- } \\
\text { protein glycosyltransferase } \\
\text { subunit STT3A }\end{array}$ & 9.86 & 0.24 & $x$ & $x$ & $\begin{array}{l}\text { protein } \mathrm{N} \text {-linked glycosylation } \\
\text { via asparagine }\end{array}$ & None \\
\hline rp6124 & $\begin{array}{l}\text { Signal recognition particle } \\
\text { receptor subunit alpha }\end{array}$ & 9.31 & 0.20 & $x$ & $\#$ & $\begin{array}{l}\text { intracellular protein transport, GTP } \\
\text { catabolic process, SRP-dependent } \\
\text { cotranslational protein targeting to } \\
\text { membrane, axonogenesis, regulation } \\
\text { of protein secretion }\end{array}$ & None \\
\hline rp6180 & $\begin{array}{l}\text { 1-acyl-sn-glycerol-3-phosphate } \\
\text { acyltransferase gamma }\end{array}$ & 9.21 & 0.49 & $1.00 \mathrm{E}-04$ & 1.00E-04 & $\begin{array}{l}\text { metabolic process, phospholipid } \\
\text { biosynthetic process }\end{array}$ & None \\
\hline rp6592 & $\begin{array}{l}\text { Sulfide:quinone oxidoreductase, } \\
\text { mitochondrial }\end{array}$ & 9.68 & 0.28 & $1.00 \mathrm{E}-04$ & 20.37 & oxidation-reduction process & $\begin{array}{l}\text { Presence of } \mathrm{C} \text {. riparius in } \\
\text { anoxic, organic sediments } \\
\text { with increased } \mathrm{H}_{2} \mathrm{~S} \text { content } \\
\text { due to effective detoxification. }\end{array}$ \\
\hline rp7097 & $\begin{array}{l}\mathrm{NADH} \text {-cytochrome b5 } \\
\text { reductase } 2\end{array}$ & 11.62 & 0.11 & $x$ & $x$ & $\begin{array}{l}\text { oxidation-reduction process, } \\
\text { lipid biosynthetic process, sterol } \\
\text { biosynthetic process }\end{array}$ & $\begin{array}{l}\text { Tolerance of C. piger to } \\
\text { increased nitrite levels due } \\
\text { to effective transformation } \\
\text { of methhaemoglobin to } \\
\text { haemoglobin. }\end{array}$ \\
\hline rp8023 & $\begin{array}{l}395 \text { ribosomal protein } L 44 \text {, } \\
\text { mitochondrial }\end{array}$ & 18.44 & 1.10 & 3.77 & 1.12 & RNA processing & $\begin{array}{l}\text { Reciprocal reproductive } \\
\text { isolation due to cytonuclear } \\
\text { incompatibilities. }\end{array}$ \\
\hline rp8328 & Calreticulin & 10.62 & 0.45 & $x$ & $x$ & $\begin{array}{l}\text { protein folding, brain morphogenesis, } \\
\text { central nervous system development, } \\
\text { peripheral nervous system } \\
\text { development, startle response, } \\
\text { olfactory behavior, locomotion } \\
\text { involved in locomotory behavior }\end{array}$ & $\begin{array}{l}\text { Tolerance of C. piger to } \\
\text { increased habitat } \\
\text { temperatures. }\end{array}$ \\
\hline
\end{tabular}

Shown are values for all alignments with a significant MKT value (FDR-corrected $p$-value
$\mathrm{x}=$ incalculable due to missing data for the four-species alignments, \# = infinite value.

$(\mathrm{Dn} / \mathrm{Ds}) /(\mathrm{Pn} / \mathrm{Ps})>1$ is an indicator of positive selection. Interspecific $\omega$ values are calculated with KaKs_Calculator using the same alignments as MKT; species-specific $\omega$ values are calculated with codeml using

four-species alignments. 
wide-spread $[41,42]$ and may result in an underestimation of intraspecific variation. However, as allele specific gene expression is due to differential DNA methylation, this may not present a substantial problem here, as Dipterans have lost the main methylation enzymes in their evolutionary history [43]. Other limitations for the use of our data are due to the experimental design. The pooling of individuals, although a common and recommended strategy for non-model organism studies aiming to identify as many alleles as possible [44], did not allow scoring individual genotypes, which in turn precluded calculations of allele frequencies. For this study, 60 individuals per species were sequenced at a mean mapping depth of 9.9 nucleotides per position for C. piger and 9.5 for C. riparius across all loci. As we have used a minimum coverage of 10 reads per position in each species for SNP detection, we have discovered only segregating alleles with a frequency over 0.25 with a probability of $>90 \%$.

\section{Interspecific patterns for the whole transcriptome}

The mean interspecific nucleotide sequence divergence of $1.53 \%$ on the cDNA level, the mean silent site rate in coding regions (dS) of 0.06 and the fact that 1,711 alignments $(21.3 \%)$ showed no differences at all, together illustrate the relatively close phylogenetic relationship between the sister species C. piger and C. riparius. Accordant calculations between two reproductively isolated 'biotypes' of the cryptic whitefly species Bemisia tabaci revealed a mean divergence of $0.83 \%$ [45], while $1.5 \%$ divergence were found between the two closely related Drosophila species D. simulans and D. sechellia [46] and $2.5 \%$ divergence between two very young sympatric crater lake cichlid fishes [47]. A recent study across transcriptomes of the copepod Tigriopus californicus even revealed higher median divergence on the interpopulation level $[2.71 \%, 17]$. On the other hand, the conservative alignment strategy taken here, with exclusion of seemingly too heterogeneous parts, may have led to a slight underestimation of the overall sequence divergence, as suggested by the $2.14 \%$ of coding sequence divergence in the vwvz/7B globin gene cluster of C. piger and C. riparius [48].

Since estimations of the species divergence time of C. piger and C. riparius based on large amounts of sequence data are lacking to date, the overall synonymous substitution rate of the ORFs was used for a rough molecular clock calculation. Drosophilids are the bestanalysed dipteran system concerning molecular clock analyses with rates of synonymous substitution per synonymous site per million years of 0.016 [49], 0.015 [50], and 0.011 [51]. Transferring these brachyceran rates to the nematocerans $C$. piger and C. riparius resulted in an estimation of the species splitting event about 1.3 to
1.8 million years ago, which translates to a multiple number of generations since divergence in this multivoltine species. This time frame clearly illustrates the existing potential for evolutionary processes to shape the species' genomes.

\section{Number of genes identified}

The number of genes found in the larval transcriptomes of $C$. piger $(6,322)$ and C. riparius $(5,705)$ is roughly a third to half the number of genes found in the genomes of the nematocerans Anopheles gambiae [ $\mathrm{n}=13,683,52]$, Aedes aegypti $[\mathrm{n}=15,419,53]$, Culex quinquefasciatus [n $=18,883,54]$ and the dipteran Drosophila melanogaster $[\mathrm{n}=13,379,55]$. The discrepancy is explained by the fact that certainly not all genes are expressed in the L4 stage of the midges and only a single stressor (heat) was applied. For example, Drosophila larval stages were shown to express only 4,900 to 7,000 different mRNAs at once [56]. Moreover, using non-normalised cDNA libraries likely resulted in missing genes with low expression levels.

\section{Global patterns of gene evolution}

The $\alpha$ statistics showed approximately a quarter (25.1\%) of the 18,115 amino acid substitutions to be driven by positive selection. This finding is close to the average of $30.1 \%$ found by Eyre-Walker in a meta-study of 25 interspecies comparisons [5]. In another meta-study Fay reported that $47 \%$ of 38 analysed species showed signs of positive selection, often affecting ca. $40 \%$ of all nonsynonymous substitutions [57]. Among other factors, the level of adaptive evolution was shown in the former study to positively correlate with effective population size [5]. Bayesian coalescence analysis of published mitochondrial COI sequences estimated the effective mitochondrial population size to be quite high with $1.8 \times 10^{7}$ (c.f. $1.7 \times 10^{6}-8.0 \times 10^{7}$ ) for C. piger and $3.1 \times 10^{6}$ (c.f. $1.3 \times 10^{5}-1.4 \times 10^{7}$ ) for C. riparius, respectively [29]. An average $\mathrm{F}_{\mathrm{ST}}$ of 0.027 for $C$. piger and 0.046 for $C$. riparius indicates little among population differentiation in both species [29]. Drift within both species thus seems to be a minor driving force here, a circumstance rendering natural selection particularly effective [58].

\section{Inference of positive selection from polymorphism data}

For the detection of positive selection on the level of individual genes, most reliable results can be gained from the MK test, which is more robust to demographic biases than, e.g. the popular $\mathrm{dN} / \mathrm{dS}$ method $[59,60]$. However, the MK approach necessitated a more than $80 \%$ reduction of the data set, due to the high sequence similarity between the sister species. Of the remaining 715 genes used to calculate MK statistics, 11 (1.5\%) showed significant signs of positive selection. 
This proportion of $1.5 \%$ of positively selected candidate genes inferred with the MKT appears rather moderate. This is especially so, since the genes expressed in the final larval stage of chironomids should be particularly prone to increased diversifying selection compared to the rest of the genome, because this is the phase in the life of the midges during which the competition (e.g. for space and food) is potentially most severe among species [61]. A recent genomic comparison of congeneric shallow and deep sea urchins indeed confirms that in particular genes expressed in the ecologically divergent life cycle stage show increased signatures of positive selection [15]. The chironomid L4 transcriptomes may thus perhaps even overestimate the impact of adaptive evolution on the entire coding genome. On the other hand, the MKT is rather conservative and positive selection having occurred early in divergence may have lost its signature [62].

\section{Ecological implications of positively selected genes}

Although a sign of positive selection alone is only the first step in defining the genetic basis of ecological differentiation, such analyses are able to deliver promising potential candidate genes [63]. Among the eleven genes with significant signs of positive selection in the MKT, eight could be functionally annotated. Five of these genes suggest a correlation either to observed or so far unrecognised ecological differences between $C$. piger and C. riparius or to the evolution of reproductive isolation.

One of them, calreticulin, is a chaperone involved in protein folding and rejection of misfolded proteins in the endoplasmic reticulum [64]. It might thus be linked to the different maximum temperatures in the respective habitats of $C$. riparius and C. piger [29], as increased temperature destabilises and degrades proteins [65].

The NADH-cytochrome b5 reductase 2 is also known as methemoglobin reductase, which describes its main function, the reduction of methaemoglobin to haemoglobin [66]. One of the main environmental agents causing the formation of detrimental methaemoglobin are nitrate/nitrite [67], thus matching the observed and experimentally confirmed differential adaptation of the chironomid sister species to environmental nitrite concentrations [29,36]. An increased efficiency due to positive selection in converting methaemoglobine would certainly explain the higher nitrite tolerance of $C$. piger and thus identify a genomic basis for the observed niche difference. Because chironomids possess more than 40 different globins $[68,69]$ which can make up as much as $90 \%$ of the last instar's total hemolymph protein [48,70], this might be of particular importance. Unfortunately, the methemoglobin reductase was not found in the dataset of all four taxa used in the branch-specific test, which precludes the inference of the direction of selection.
The branch-specific $\omega$ for plasma membrane calciumtransporting ATPase 3 (PMCA3) revealed positive selection on the $C$. piger branch $(\omega=9.75)$, as opposed to a neutral evolution of this gene in $C$. riparius $(\omega=0.08)$. PMCA3 is responsible for the removal of calcium out of the cell [71]. Interestingly, Pfenninger and Nowak [29] demonstrated conductivity in general and $\mathrm{CaCO}_{3}$ concentrations in particular to be significantly correlated to the relative abundance of $C$. piger and $C$. riparius, with the latter preferring lower concentrations, thus lending ecological plausibility to PMCA3 as a candidate gene for positive selection.

Besides proposing candidate genes for the known ecological differences between $C$. riparius and $C$. piger, the reverse ecology approach of studying whole transcriptomes also yields novel gene candidates with plausible relevance to the ecology and evolution of the sister taxa:

Sulfide:quinone oxidoreductase is involved in hydrogen sulfide detoxification [72]. Hydrogen sulfide is produced by microorganisms under anaerobic conditions, being highly toxic to most metazoans. The prevalence of C. riparius in anaerobic habitats like sewage sludge [34] might therefore at least partly be based on higher hydrogen sulfide tolerance. In accordance with this hypothesis is the high $\omega$ detected on the C. riparius branch (20.37) for sulfide:quinone oxidoreductase in contrast to the $C$. piger branch $\left(1 \mathrm{e}^{-4}\right)$, suggesting adaptation to increased $\mathrm{H}_{2} \mathrm{~S}$ levels in sediments of eutrophic water.

The nuclear encoded 39S ribosomal protein L44 interacts closely with mitochondrially encoded rRNA molecules [73], thus providing the basis for cytonuclear coevolution, in which structural changes in one partner (usually the faster evolving mitochondrial genome) provide the stage for selection-driven compensatory changes in the interaction partner [74]. Intriguingly, the branch specific analysis shows positive selection along the branches of both species. If the resulting changes in protein structure led to incompatibilities with the mitochondrial rRNA of the respective other species in the formation of mitochondrial ribosomes, they may at least partially explain the observed reproductive isolation in the wild [75]. Cytonuclear incompatibilities indeed have been shown to confer reproductive isolation among other insects species [76].

\section{Conclusions}

In accordance with recent meta-studies [5,57] on the genome-wide prevalence of positive selection in insects, the data presented here argue that such Darwinian processes are likely to have played an important role in the divergence of Chironomus piger and C. riparius. Several gene loci showing signatures of directional or disruptive positive selection can explain observed ecological characteristics of niche divergence. In addition, the reverse 
ecology approach pointed towards a new hypothesis with respect to the higher anaerobic tolerance of $C$. riparius. The signs of positive selection on a sulfide:quinone oxidoreductase gene in this species suggest the possibility that this adaptation depends at least partly on a higher $\mathrm{H}_{2} \mathrm{~S}$-tolerance.

Obviously, the above hypotheses, based solely on bioinformatic analysis of transcriptome sequences, need further investigations, in particular ecological experiments and functional protein assays, to functionally link the proposed candidate genes to the observed traits. In addition, there are likely several, if not many other fixed functional differences among the sister species, which affect protein structure and gene expression and thereby determine their respective niches. The presented study is therefore but a first step to fully understand the genomic basis of the ecological differences between $C$. piger and C. riparius. However, since many of the known ecological differences are plausibly mirrored by patterns of sequence evolution, the taken approach appears to be a valuable starting point to dig deeper into the genomic details of niche segregation.

\section{Methods}

The Chironomus riparius and C. piger specimen used in this study were taken from laboratory cultures originally collected in 2009 from the field in south-western Germany as described in Nemec et al. [36]. The species were kept in relatively large ( $>400$ individuals) populations for about 3-4 generations prior to the present study. In a previous study, populations of this size kept for more than 10 generations under identical conditions showed no significant reduction of genetic diversity due to drift [77]. Freshly hatched larvae were reared at $20^{\circ} \mathrm{C}$ and $27^{\circ} \mathrm{C}$ in climate chambers with 16 hours lighting per day and fed ad libitum on commercial flake food for aquarium fish. The two different conditions were chosen because the resulting data was planned to be used for a study on differential gene expression and play a minor role here. On attaining larval stage four (L4), 30 larvae per treatment and species were shock-frozen at $-80^{\circ} \mathrm{C}$.

\section{RNA isolation, library preparation and sequencing}

RNA isolation with Qiagen RNeasy Mini Kit (Qiagen) from the 30 whole larvae per treatment and species was followed by DNase digestion and purification of mRNA with Invitrogen Dynabeads (Invitrogen). Subsequently, four cDNA libraries, barcoded with multiplex identifier adaptors, were created using the cDNA Rapid Library Preparation Method Manual (Roche). Libraries were not normalized since this data was intended for use in a further gene expression study. After titration, each library was sequenced on half a titanium plate on a Roche 454 GS FLX system (Roche) according to the manufacturers' instructions. All sequences were deposited in the NCBI Sequence Read Archive (accession numbers SRR834934, SRR835079, SRR834592, SRR834593). Sequences were processed to remove adaptors, low quality sequence parts (at least an average quality score of 20 for the last ten bp), and sequences below 50 bp prior to assembly.

\section{Assembly and annotation}

Filtered reads were pooled per species and assembled de novo using the CLC Genomics Workbench 4.9 (CLC Bio, Aarhus, Denmark) with default settings (word size $=20$, bubble size $=50$ ). After assembly all reads were mapped back against the contig sequences for quality control and identification of variable sites (see below).

All obtained contigs were compared to the Swiss-Prot database using the BLASTx algorithm [78] with a cut-off E-value of $\leq 1 \mathrm{e}^{-10}$. Functional annotation was performed using the terminology provided by the Gene Ontology (GO) Consortium [79] in the category Biological Process (BP). This category "refers to a biological objective to which the gene or gene product contributes" [79] and therefore allows most informative linkage to ecological differences. The Swiss-Prot accession number of the best local alignment was used to annotate the respective gene with the associated GO terms. The Uniprot GO annotation file (v2.0) was obtained from the download section of geneontology.org.

\section{Obtaining high quality alignments of orthologous sequences of $C$. piger and $C$. riparius}

Protein sequences were obtained by predicting open reading frames (ORFs) with the Python program ORFPredictor (unpublished software by L. Wissler, available upon request by the authors). ORFPredictor uses the best local alignment from the BLASTx searches described above and searches the respective ORF from that starting point. For all contigs without BLAST hit, ORFPredictor outputs the longest contiguous closed ORF above the set minimum of 30 amino acids. Everything but the ORF sequences was discarded for downstream analyses. To obtain alignments of orthologous genes, the protein sequences were clustered using orthoMCL 2.0 [80] with standard settings. To remove paralogs, the sequences of all clusters with at least one sequence of each species were aligned using T-Coffee 8.99 [81]. For each cluster only the sequence of each species with the highest similarity to the sequence of the other species was used for further analyses. Pair-wise alignments were created using MUSCLE 3.8.31 [82] with standard settings. Those final protein alignments were (re)converted to the respective nucleotide alignments using PAL2NAL 13.0 [83], drawing on the respective nucleotide sequences after ORF-prediction. The nucleotide alignments were trimmed to the same length in both 
species. Visual inspection of the alignments showed the ends to frequently contain frame shifts, mainly due to differences in homopolymer length in 454 Chironomus sequences. Consequently, the nucleotide alignments were further trimmed by $15 \mathrm{bp}$ at each end. As an additional quality control step, all alignments with a pairwise p-distance $>0.1$ between the two Chironomus species were excluded. This highly conservative filtering to remove residual poor alignments was chosen as it has been shown that even the intergenic regions of $C$. piger and $C$. riparius show an overall sequence identity of $90.8 \%$ [48].

\section{Counting polymorphic and divergent sites}

Substitution counts were obtained from the output of KaKs_Calculator 1.2 [84], SNP detection was performed using the accordant tool in the CLC Genomics Workbench and the respective mappings of the aligned contigs. Only SNPs with a minimum mapping depth of ten, two alleles and at least two occurrences of the rarer allele were considered. Any SNP with a quality score below 20 and any with more than three surrounding bases with quality scores below 15 was ignored. SNPs then got classified as silent/ non-silent using selfwritten scripts. SNP-counts for both species were added up per gene.

\section{Measurement of sequence evolution for the whole transcriptome}

Evidence for the transcriptome-wide prevailing mode of selection between two species can be gained by estimating $\alpha$, the average proportion of interspecific amino-acid substitutions which are driven by adaptive evolution $[85,86]$. The calculation was performed using the method described by Smith and Eyre-Walker [86] and implemented in the DoFE software package 3.0 [87]. The analysis was based on the counts for nonsynonymous and synonymous substitutions, non-silent and silent polymorphisms and non-synonymous and synonymous positions per gene of the 8,031 two-species alignments. The confidence interval for $\alpha$ was obtained by 1,000 bootstraps with randomly selected genes. As slightly deleterious mutations contribute more to polymorphisms than to divergence, the SNPs were then classified by the frequency of their rarer allele into low frequency SNPs $(<5 \%)$, moderate frequency SNPs (5$15 \%)$, and common SNPs (15-50\%) following Fay et al. [88]. Afterwards, the calculation of $\alpha$ was repeated under exclusion of low frequency SNPs and low+moderate frequency SNPs, respectively.

\section{McDonald-Kreitman tests}

The McDonald-Kreitman test [89] identifies patterns of sequence evolution by comparing synonymous/non- synonymous divergence versus silent/non-silent polymorphisms. It is very robust to different demographic factors $[59,60]$ and does not suffer from inherent problems of $\omega$ analyses concerning nonlinear correlations of two randomly distributed variables [90]. The MKT itself depends on the assumption that $D_{n} / D_{s}>P_{n} / P_{s}$ indicates an excess of adaptive amino acid substitutions and thus, positive selection. No input value must be zero. The distribution across the four classes is then tested for independence on a $2 \times 2$ contingency table using an appropriate statistic ( $\chi^{2}$ test with false discovery rate (FDR) correction for this study).

\section{Inferring the direction of positive selection}

To distinguish whether positive selection acted directionally on the branch leading to the one or the other species for the genes identified by MKT, a likelihood approach depending on phylogenetic alignments was used. Protein sequences from the phylogenetically closest genome sequenced organisms Aedes aegypti (PEPTIDES-AaegL1.2) and Culex quinquefasciatus (PEPTIDES-CpipJ1.2) were downloaded from VectorBase (vectorbase.org). The predicted ORFs for $C$. piger and $C$. riparius specified above were also used for the subsequent analyses. Clusters obtained by OrthoMCL as described above were split into two groups, according to phylogenetic relatedness: $C$. riparius and $C$. piger as non-biting midges and $A$. aegypti and $C$. quinquefasciatus as biting midges. Pair-wise MUSCLE alignments of these sub-clusters were then merged with the same program and re-translated as described above, additionally using the nucleotide sequences of C. quinquefasciatus (TRANSCRIPTS-CpipJ1.2) and $A$. aegypti (TRANSCRIPTS-AaegL1.2) from VectorBase. Trimming was performed as described in the upper part.

Tests on positive selection were performed by applying the codeml algorithm from the PAML program package $4.4[91,92]$ to the four-species alignments. Some models in codeml allow discrete $\omega$ values for different taxa in one analysis, providing valuable information about the species' contribution to divergent evolution. Calculation of global $\omega$ values using the one ratio model was followed by calculation of branch-specific $\omega$ using the free ratio model. As the free ratio model is very parameter-rich and therefore prone to biases, only alignments with significantly better likelihood scores $\left(\mathrm{X}^{2}, \mathrm{p}=\right.$ $0.05)$ for the more sophisticated free ratio model were considered for subsequent summary. Alignments with $\mathrm{dS}>1$ on the chironomid branches were discarded.

\section{Additional file}

Additional file 1: GO annotation of transcripts. Representation of the GO terms from the category Biological Process across the Blastx annotated transcripts. Percentages are based on the number of genes successfully 
annotated per species. Shown are all GO terms associated to at least $1.5 \%$ of all transcripts.

\section{Competing interests}

The authors declare that they have no competing interests.

\section{Authors' contributions}

HS carried out the experiments and data analysis, participated in laboratory and bioinformatics work and drafted the manuscript. BG carried out bioinformatics work from orthology assignment to final alignments. BF participated in laboratory work and the study's design and coordination. TH conceived the study, its design and coordination and helped to draft the manuscript. MP conceived the study, its design and coordination and drafted the manuscript. All authors read and approved the final manuscript.

\section{Acknowledgements}

We are grateful to three anonymous reviewers for their exceptionally constructive comments on a previous version of the manuscript. This work was supported by the research funding programme "LOEWE - LandesOffensive zur Entwicklung Wissenschaftlich-ökonomischer Exzellenz" of Hesse's Ministry of Higher Education, Research, and the Arts. TH gratefully acknowledges funding by the University of Mainz Center for Computationa Sciences (SRFN).

We want to thank Mathias Weber and Benjamin Rieger for writing several useful Perl scripts and Lothar Wissler for providing his programme ORFPredictor.

\section{Author details}

${ }^{1}$ Molecular Ecology Group, Biodiversity and Climate Research Centre (BiK-F) by Senckenberg Gesellschaft für Naturforschung and Goethe University, Biocampus Siesmayerstraße, 60054, Frankfurt am Main, Germany. ${ }^{2}$ Institute of Molecular Genetics, Biosafety Research and Consulting, Johannes GutenbergUniversity, Becherweg 30a, 55128, Mainz, Germany. ${ }^{3}$ Current address: Department of Evolutionary Biology, Johannes Gutenberg-University, Johannes-von-Müller-Weg 6, 55128, Mainz, Germany.

Received: 1 March 2013 Accepted: 30 May 2013

Published: 10 June 2013

\section{References}

1. Hughes AL: Looking for Darwin in all the wrong places: the misguided quest for positive selection at the nucleotide sequence level. Heredity 2007, 99(4):364-373.

2. Gillespie JH: The status of the Neutral Theory. Science 1984, 224(4650): 732-733.

3. Hahn MW: Toward a selection Theory of Molecular Evolution. Evolution 2008, 62(2):255-265.

4. Nei M, Suzuki $Y$, Nozawa M: The neutral theory of molecular evolution in the genomic era. Annu Rev Genomics Hum Genet 2010, 11:265-289.

5. Eyre-Walker A: The genomic rate of adaptive evolution. Trends Ecol Evol 2006, 21(10):569-575.

6. Kimura M: Evolutionary Rate at the Molecular Level. Nature 1968 217:624-626.

7. Kimura M: The neutral theory of molecular evolution. Cambridge, UK: Cambridge University Press; 1983

8. Ohta T: Population size and rate of evolution. J Mol Evol 1972, 1(4):305-314

9. Ohta T: The nearly neutral theory of molecular evolution. Annu Rev Ecol Syst 1992, 23:263-286.

10. Sella G, Petrov DA, Przeworski M, Andolfatto P: Pervasive natural selection in the Drosophila genome? PLoS Genet 2009, 5(6):e1000495.

11. Begun DJ, Holloway AK, Stevens K, Hillier LDW, Poh YP, Hahn MW, Nista PM Jones CD, Kern AD, Dewey CN: Population genomics: whole-genome analysis of polymorphism and divergence in Drosophila simulans. PLoS Biol 2007, 5(11):e310.

12. Shendure J, Ji H: Next-generation DNA sequencing. Nat Biotechnol 2008 , 26:1135-1145

13. Ansorge WJ: Next-generation DNA sequencing techniques. New Biotechnology 2009, 25(4):195-203.

14. Ekblom R, Galindo J: Applications of next generation sequencing in molecular ecology of non-model organisms. Heredity 2011, 107(1):1-15.
15. Oliver TA, Garfield DA, Manier MK, Haygood R, Wray GA, Palumbi SR: Wholegenome positive selection and habitat-driven evolution in a shallow and a deep-sea urchin. Genome Biol Evol 2010, 2:800-814.

16. Vera JC, Wheat CW, Fescemyer HW, Frilander MJ, Crawford DL, Hanski I, Marden JH: Rapid transcriptome characterization for a nonmodel organism using 454 pyrosequencing. Mol Ecol 2008, 17(7):1636-1647.

17. Barreto FS, Moy GW, Burton RS: Interpopulation patterns of divergence and selection across the transcriptome of the copepod Tigriopus californicus. Mol Ecol 2010, 20:560-572.

18. Meyer E, Aglyamova GV, Matz MV: Profiling gene expression responses of coral larvae (Acropora millepora) to elevated temperature and settlement inducers using a novel RNA-Seq procedure. Mol Ecol 2011, 20(17):3599-3616.

19. Price DP, Nagarajan V, Churbanov A, Houde P, Milligan B, Drake LL, Gustafson JE, Hansen IA: The fat body transcriptomes of the yellow fever mosquito aedes aegypti, pre- and post- blood meal. PLoS One 2011, 6(7):e22573

20. Li YF, Costello JC, Holloway AK, Hahn MW, Rausher M: "Reverse ecology" and the power of population genomics. Evolution 2008, 62(12):2984-2994.

21. Guryev V, Makarevitch I, Blinov A, Martin J: Phylogeny of the genus Chironomus (Diptera) inferred from dna sequences of mitochondrial cytochrome b and cytochrome oxidase I. Mol Phylogen Evol 2001, 19(1):9-21.

22. MacArthur $\mathrm{RH}$, Levins $\mathrm{R}$ : The limiting similarity, convergence and divergence of coexisting species. Am Nat 1967, 101:377-385.

23. Schneider A, Souvorov A, Sabath N, Landan G, Gonnet GH, Graur D: Estimates of positive Darwinian selection are inflated by errors in sequencing, annotation, and alignment. Genome Biol Evol 2009, 1:114-118.

24. Ellegren $\mathrm{H}$ : Evolution: natural selection in the evolution of humans and chimps. Curr Biol 2005, 15(22):R919-R922.

25. Miehlbradt J, Neumann D: Reproduktive isolation durch optische Schwarmmarken bei den sympatrischen Chironomus thummi und $\mathrm{Ch}$. piger. Behaviour 1976, 58(26):272-297.

26. Keyl HG, Strenzke K: Taxonomie und cytologie von zwei subspezies der art Chironomus thummi. Zeitschrift für Naturforschung Part B 1956 11:727-735.

27. Hägele K: Hybrid syndrome-induced postzygotic reproductive isolation: a second reproduction barrier in Chironomus thummi (Diptera, Chironomidae). J Zool Syst Evol Res 1999, 37(4):161-164.

28. Strenzke K: Die systematische und ökologische Differenzierung der Gattung Chironomus. Ann Entomol Fenn 1960, 26:111-139.

29. Pfenninger M, Nowak C: Reproductive isolation and ecological niche partition among larvae of the morphologically cryptic sister species Chironomus riparius and C. piger. PLoS One 2008, 3(5):e2157.

30. Pfenninger M, Nowak C, Kley C, Steinke D, Streit B: Utility of DNA taxonomy and barcoding for the inference of larval community structure in morphologically cryptic Chironomus (Diptera) species. Mol Ecol 2007. 16(9):1957-1968

31. Oliver D: Life history of the Chironomidae. Annu Rev Entomol 1971, 16:211-230

32. Pinder L: Biology of freshwater Chironomidae. Annu Rev Entomol 1986, 31:1-23.

33. Gower AM, Buckland PJ: Water quality and the occurence of Chironomus riparius Meigen (Diptera: Chironomidae) in a stream receiving sewage fluent. Freshwat Biol 1978, 8:153-164.

34. Neumann D: Die anaerobiose-toleranz der larven zweier subspezies von Chironomus thummi. Zeitschrift fuer vergleichende Physiologie 1962, 46:150-162.

35. Scharf BW: Experimentell-ökologische untersuchungen an Chironomus thummi und Chironomus piger (Diptera, Chironomidae). Arch Hydrobiol 1973, 72(2):225-244

36. Nemec S, Heß M, Nowak C, Pfenninger M: Experimental confirmation of field observed niche segregation in a species pair of non-biting midges. Hydrobiologia 2012. doi:10.1007/s10750-012-1074-4

37. Mardis ER: Next-generation DNA sequencing methods. Annu Rev Genomics Hum Genet 2008, 9:387-402.

38. Balzer S, Malde K, Lanzén A, Sharma A, Jonassen I: Characteristics of 454 pyrosequencing data - enabling realistic simulation with flowsim. Bioinformatics 2010, 26(18):i420-i425.

39. Gilles A, Meglécz E, Pech N, Ferreira S, Malausa T, Martin JF: Accuracy and quality assessment of $454 \mathrm{GS}-\mathrm{FLX}$ Titanium pyrosequencing. BMC Genomics 2011, 12(1):245. 
40. Knight JC: Allele-specific gene expression uncovered. Trends Genet 2004, 20(3):113-116.

41. Tycko B, Morison IM: Physiological functions of imprinted genes. J Cell Physiol 2002, 192(3):245-258

42. Lo HS, Wang Z, Hu Y, Yang HH, Gere S, Buetow KH, Lee MP: Allelic variation in gene expression is common in the human genome. Genome Res 2003, 13(8):1855-1862.

43. Glastad KM, Hunt BG, Yi SV, Goodisman MAD: DNA methylation in insects: on the brink of the epigenomic era. Insect Mol Biol 2011, 20(5):553-565.

44. Futschik A, Schlötterer C: The next generation of molecular markers from massively parallel sequencing of pooled DNA samples. Genetics 2010, 186(1):207-218.

45. Wang XW, Luan JB, Li JM, Su YL, Xia J, Liu SS: Transcriptome analysis and comparison reveal divergence between two invasive whitefly cryptic species. BMC Genomics 2011, 12(1):458

46. McDermott SR, Kliman RM: Estimation of isolation times of the island species in the Drosophila simulans complex from multilocus DNA sequence data. PLoS One 2008, 3(6):e2442

47. Elmer KR, Fan S, Gunter HM, Jones JC, Boekhoff S, Kuraku S, Meyer A: Rapid evolution and selection inferred from the transcriptomes of sympatric crater lake cichlid fishes. Mol Ecol 2010, 19:197-211.

48. Trewitt PM, Luhm RA, Samad F, Ramakrishnan S, Kao W-Y, Bergtrom G: Molecular evolutionary analysis of the YWVZ/7B globin gene cluster of the insect Chironomus thummi. J Mol Evol 1995, 41:313-328.

49. Sharp PM, Li WH: On the rate of DNA sequence evolution in Drosophila. J Mol Evol 1989, 28(5):398-402.

50. Rowan RG, Hunt JA: Rates of DNA change and phylogeny from the DNA sequences of the alcohol dehydrogenase gene for five closely related species of Hawaiian Drosophila. Mol Biol Evol 1991, 8(1):49-70.

51. Tamura K, Subramanian S, Kumar S: Temporal patterns of fruit fly (Drosophila) evolution revealed by mutation clocks. Mol Biol Evol 2004, 21(1):36-44.

52. Holt RA, Subramanian GM, Halpern A, Sutton GG, Charlab R, Nusskern DR, Wincker $P$, Clark AG, Ribeiro JMC, Wides R: The genome sequence of the malaria mosquito Anopheles gambiae. Science 2002, 298(5591):129.

53. Nene V, Wortman JR, Lawson D, Haas B, Kodira C, Tu Z, Loftus B, Xi Z, Megy K, Grabherr M, et al: Genome sequence of Aedes aegypti, a major arbovirus vector. Science 2007, 316(5832):1718-1723.

54. Arensburger P, Megy K, Waterhouse RM, Abrudan J, Amedeo P, Antelo B, Bartholomay L, Bidwell S, Caler E, Camara F, et al: Sequencing of Culex quinquefasciatus establishes a platform for mosquito comparative genomics. Science 2010, 330(6000):86-88.

55. Adams MD, Celniker SE, Holt RA, Evans CA, Gocayne JD, Amanatides PG, Scherer SE, Li PW, Hoskins RA, Galle RF: The genome sequence of Drosophila melanogaster. Science 2000, 287(5461):2185.

56. Levy LS, Manning JE: Messenger RNA sequence complexity and homology in developmental stages of Drosophila. Dev Biol 1981, 85(1): 141-149.

57. Fay JC: Weighing the evidence for adaptation at the molecular level. Trends Genet 2011, 27(9):343-349.

58. Wright S: Evolution in Mendelian populations. Genetics 1931, 16(2):97.

59. Nielsen R: Molecular signatures of natural selection. Annu Rev Genet 2005, 39:197-218

60. MacCallum C, Hill E: Being positive about selection. PLoS Biol 2006, 4(3): 293-295.

61. Tokeshi M: Species interactions and community structure. In The Chironomidae: the Biology and Ecology of Non-Biting Midges. Edited by Armitage PD, Cranston PS, Pinder LCV. London, UK: Chapman \& Hall 1995:297-335.

62. Hudson RR, Kreitman M, Aguadé M: A test of neutral molecular evolution based on nucleotide data. Genetics 1987, 116(1):153-159.

63. Jensen JD, Wong A, Aquadro CF: Approaches for identifying targets of positive selection. Trends Genet 2007, 23(11):568-577.

64. Nakatsukasa K, Brodsky JL: The recognition and retrotranslocation of misfolded proteins from the endoplasmic reticulum. Traffic 2008, 9(6): 861-870.

65. Goldberg AL: Protein degradation and protection against misfolded or damaged proteins. Nature 2003, 426(6968):895-899.

66. Yubisui T, Miyata T, Iwanaga S, Tamura M, Yoshida S, Takeshita M, Nakajima $\mathrm{H}$ : Amino acid sequence of $\mathrm{NADH}$-cytochrome b5 reductase of human erythrocytes. J Biochem 1984, 96(2):579-582.
67. Shuval HI, Gruener N: Epidemiological and toxicological aspects of nitrates and nitrites in the environment. Am J Public Health 1972, 62(8): 1045-1052.

68. Green BN, Kuchumov AR, Hankeln T, Schmidt ER, Bergtrom G, Vinogradov $\mathrm{SN}$ : An electrospray ionization mass spectrometric study of the extracellular hemoglobins from Chironomus thummi thummi. Biochimica et Biophysica Acta (BBA) - Protein Structure and Molecular Enzymology 1998, 1383(1):143-150

69. Hankeln T, Amid C, Weich B, Niessing J, Schmidt ER: Molecular evolution of the globin gene cluster $\mathrm{E}$ in two distantly related midges. Chironomus pallidivittatus and C. thummi thummi. J Mol Evol 1998, 46(5):589-601.

70. Bergtrom G, Laufer $H$, Rogers R: Fat body: a site of hemoglobin synthesis in Chironomus thummi (diptera). J Cell Biol 1976, 69(2):264-274.

71. Carafoli E: The Ca2+ pump of the plasma membrane. J Biol Chem 1992 267(4):2115-2118

72. Brito JA, Sousa FL, Stelter M, Bandeiras TM, Vonrhein C, Teixeira M, Pereira $\mathrm{MM}$, Archer M: Structural and functional insights into sulfide: quinone oxidoreductase. Biochemistry 2009, 48(24):5613-5622.

73. Benne R, Sloof P: Evolution of the mitochondrial protein synthetic machinery. Bio Systems 1987, 21(1):51.

74. Rand DM, Haney RA, Fry AJ: Cytonuclear coevolution: the genomics of cooperation. Trends Ecol Evol 2004, 19(12):645-653.

75. Chou JY, Leu JY: Speciation through cytonuclear incompatibility: insights from yeast and implications for higher eukaryotes. Bioessays 2010, 32(5): 401-411.

76. Ellison CK, Niehuis O, Gadau J: Hybrid breakdown and mitochondrial dysfunction in hybrids of Nasonia parasitoid wasps. J Evol Biol 2008, 21(6): 1844-1851.

77. Nowak C, Vogt C, Pfenninger M, Schwenk K, Oehlmann J, Streit B, Oetken M: Rapid genetic erosion in pollutant-exposed experimental chironomid populations. Environ Pollut 2009, 157(3):881-886.

78. Altschul SF, Gish W, Miller W, Myers EW, Lipman DJ: Basic local alignment search tool. J Mol Biol 1990, 215(3):403-410.

79. Ashburner M, Ball CA, Blake JA, Botstein D, Butler H, Cherry JM, Davis AP, Dolinski K, Dwight SS, Eppig JT: Gene Ontology: tool for the unification of biology. Nat Genet 2000, 25(1):25.

80. Li L, Stoeckert CJ, Roos DS: OrthoMCL: identification of ortholog groups for eukaryotic genomes. Genome Res 2003, 13(9):2178-2189.

81. Notredame C, Higgins DG, Heringa J: T-Coffee: A novel method for fast and accurate multiple sequence alignment. J Mol Biol 2000, 302(1):205-217.

82. Edgar RC: MUSCLE: multiple sequence alignment with high accuracy and high throughput. Nucleic Acids Res 2004, 32(5):1792-1797.

83. Suyama M, Torrents D, Bork P: PAL2NAL: robust conversion of protein sequence alignments into the corresponding codon alignments. Nucleic Acids Res 2006, 34(suppl 2):W609-W612.

84. Zhang Z, Li J, Zhao X-Q, Wang J, Wong GK-S, Yu J: KaKs_Calculator: calculating $\mathrm{Ka}$ and $\mathrm{Ks}$ through model selection and model averaging. Genomics, Proteomics \& Bioinformatics 2006, 4(4):259-263.

85. Charlesworth $B$ : The effect of background selection against deleterious mutations on weakly selected, linked variants. Genet Res 1994, 63(3):213228

86. Smith NG, Eyre-Walker A: Adaptive protein evolution in Drosophila. Nature 2002, 415(6875):1022-1024.

87. Stoletzki N, Eyre-Walker A: Estimation of the neutrality index. Mol Biol Evol 2011, 28(1):63-70

88. Fay JC, Wyckoff GJ, Wu C-I: Positive and negative selection on the human genome. Genetics 2001, 158(3):1227-1234.

89. McDonald $J \mathrm{H}$, Kreitman M: Adaptive protein evolution at the Adh locus in Drosophila. Nature 1991, 351(6328):652-654.

90. Wolf JBW, Künstner A, Nam K, Jakobsson M, Ellegren H: Nonlinear dynamics of nonsynonymous (dN) and synonymous (dS) substitution rates affects inference of selection. Genome Biol Evol 2009, 1:308-319.

91. Yang Z: PAML: a program package for phylogenetic analysis by maximum likelihood. CABIOS 1997, 13(5):555.

92. Yang Z: PAML 4: phylogenetic analysis by maximum likelihood. $\mathrm{Mol}$ Biol Evol 2007, 24(8):1586-1591.

doi:10.1186/1471-2164-14-384

Cite this article as: Schmidt et al:: Genomic basis of ecological niche divergence among cryptic sister species of non-biting midges. BMC Genomics 2013 14:384. 